Nouvelles perspectives en sciences sociales

\title{
Observatoires de la vie étudiante des universités contre Portail étudiant du Ministère : conflit statistique, territorial ou organisationnel?
}

\section{Geoffrey Carrère et Nicolas Malinowski}

Volume 3, numéro 2, mars 2008

URI : https://id.erudit.org/iderudit/602477ar

DOI : https://doi.org/10.7202/602477ar

Aller au sommaire du numéro

Éditeur(s)

Prise de parole

ISSN

1712-8307 (imprimé)

1918-7475 (numérique)

Découvrir la revue

Citer cet article

Carrère, G. \& Malinowski, N. (2008). Observatoires de la vie étudiante des universités contre Portail étudiant du Ministère : conflit statistique, territorial ou organisationnel? Nouvelles perspectives en sciences sociales, 3(2), 83-111. https://doi.org/10.7202/602477ar
Résumé de l'article

En France, l'université a fait longtemps l'objet d'un désintérêt sociologique, et ce, contrairement à celle des pays anglo-saxons. Cet article participe donc à l'ouverture de la boîte noire universitaire par une réflexion au coeur de l'actualité politique relative à l'autonomie et à l'adaptabilité de l'université au monde du travail. Afin de répondre à cette problématique contemporaine, le Ministère de l'enseignement supérieur et de la recherche a proposé la création d'un nouveau site Web, le Portail étudiant, auquel s'opposent les observatoires de la vie étudiante (OVE). L'analyse de ce conflit d'ordre méthodologique soulève un questionnement plus large sur la prégnance de la dimension territoriale dans la construction des indicateurs statistiques réalisés par les OVE. 


\section{Observatoires de la vie étudiante des universités contre Portail étudiant du Ministère : conflit statistique, territorial ou organisationel ?}

Geoffrey CARRÈRE

CIRESS-LEREPS

Université des Sciences Sociales de Toulouse, Toulouse 1 et département de sociologie de L'Université Laval, Québec

Nicolas MALINOWSKI

CIRESS-LEREPS

Université des Sciences Sociales de Toulouse, Toulouse 1 et Multiversité Mundo Real-Edgar Morin, Hermosillo, Mexique

"L'Université doit-elle s'adapter à la société ou la société doit-elle s'adapter à l'Université ? Il y a complémentarité et antagonisme entre les deux missions, s'adapter à la société et adapter à soi la société $[\ldots]^{1}$ ». Cette réflexion soulevée par Edgar Morin en 1999 nous semble encore d'actualité, 'eu égard aux nombreux débats sur la loị relative aux libertés et responsabilités des universités du 10 août $2007^{2}$. La question de l'autonomie, mais également celle de l'adaptabilité de l'institution

Edgar Morin, La tête bien faite, Paris, Seuil, 1999, p. 94.

2 La Direction de l'évaluation, de la Prospective et de la Performance du ministère de l'éducation nationale a pour objectif la mise en place d'étude sur les pratiques pédagogiques. Elle les réalise seule ou en collaboration avec les inspections générales, des équipes de recherche, des organismes extérieurs nationaux ou internationaux. 
universitaire à la société et, plus précisément, au monde du travail est l'une des problématiques politiques actuelles. Le projet Insertion professionnelle des sortants de l'enseignement supérieur (IPSES), lancé en mai 2006 par le Ministère de l'enseignement supérieur et de la recherche (MESR) à la suite des manifestations étudiantes liées à la réforme du contrat première embauche (CPE), se présente comme une tentative de réponse politique à ces problèmes.

Ce projet s'incarne notamment par la création d'un nouveau site Web à caractère informatif, le Portail étudiant. L'objectif de ce portail consiste à regrouper de manière exhaustive les informations relatives aux brevets de technicien supérieur (BTS), aux licences professionnelles et aux différents cursus de chaque université, afin de faciliter, pour l'étudiant, l'élaboration d'un projet d'étude cohérent. Ces informations portent autant sur l'accueil des étudiants, sur les projets d'étude à l'étranger, sur les conditions de vie étudiante (financement, santé, logement), que sur l'insertion professionnelle et les taux de réussite de chaque université. Les collectes d'informations sont réalisées respectivement par la Direction de l'évaluation de la prospective et de la performance (DEPP A1), la Direction générale de l'enseignement supérieur (DGES), le Service commun universitaire d'information et d'orientation (SCUIO) et les services d'information des universités.

Six mois après le lancement du projet IPSES, seuls quatorze établissements sur cinquante avaient accepté de remplir les grilles statistiques proposées par le portail. On assiste ainsi à l'expression d'une opposition notable à ce projet de la part des observatoires de la vie étudiante (OVE). Que sont les OVE? Il s'agit d'observatoires templissant une double activité : élaborer le projet universitaire de leur établissement et préparer le plan régional de développement des formations (PRDF) en partenariat avec le rectorat, le Conseil régional et les autres universités locales. L'opposition des OVE au projet IPSES s'articule autour de trois arguments principaux : les inégalités en termes de comparabilité des statistiques produites, les antagonismes méthodologiques entre les OVE et le Ministère et la non-prise en compte de l'hétérogénéité des contextes dans lesquels s'inscrivent leurs études.

Ces insuffisances d'ordre méthodologique sont à la base d'un conflit qui présente un double enjeu. Premièrement, celui de la connaissance scientifique du milieu étudiant. Le Ministère semble affirmer la volonté d'harmoniser et de centraliser les informations locales. Deuxjèmement, 
celui du traitement et de l'interprétation des données statistiques recueillies par les OVE, considérés comme trop laxistes. Ce problème soulève alors la question de l'encastrement territorial des indicateurs statistiques. Dans un même mouvement, la problématique de la diffusion des résultats est essentielle, car elle constitue l'un des facteurs explicatifs de la revendication des spécificités locales motivée par la remise en cause du contrôle des experts locaux sur les indicateurs statistiques.

Ce contexte de conflit méthodologique entre les OVE et le Ministère, au travers du projet IPSES, fournit un terrain sociologique intéressant quant à l'enjeu de la défense des particularités locales (conjoncture économique, marché du travail régional). La volonté de défense des connaissances locales a favorisé la création du réseau des observatoires de la vie étudiante (ROVE), en tant que première association nationale des OVE. Cette association a pour objectif essentiel d'établir un contrepouvoir notable dans le dialogue avec le Ministère. Ainsi, l'objectif de notre article est-il double. D'une part, il s'agit de s'interroger sur le processus sociotechnique de construction des indicateurs statistiques de l'insertion étudiante. En ce sens, la statistique n'est pas envisagée comme un champ disciplinaire produisant des données " objectives", mais bien comme une discipline influencée et construite par son environnement, encastrée territorialement. D'autre part, la problématique de la diffusion des résultats scientifiques, à l'origine de la mutation du désaccord méthodologique en repositionnement organisationnel, se traduisant par une forte revendication des spécificités locales, fournit le cas manifeste d'un conflit s'inscrivant dans un rapport savoir/pouvoir.

Afin de mener à bien notre étude nous avons étudié l'OVE de l'Université de Toulouse 1, créé, en 1992, en tant que service directement rattaché à la Présidence de l'université. D'un point de vue formel, l'équipe se compose de quatre membres permanents titulaires de poste. Le directeur, également responsable d'une maitrise professionnelle (Ingénierie de la formation et des systèmes d'emploi) et chargé d'une chaire à l'UNESCO sur la formation appliquée au développement local $^{3}$, a pour tâche de superviser et de coordonner les productions

3 Organisation des Nations Unies pour l'éducation, la science et la culture, http://portal.unesco.org/education/fr, consulté le 14 décembre 2006. 
statistiques de ses deux responsables de pôles. Il a également sous sa tutelle une personne remplissant une activité de secrétariat. Les deux responsables sont, l'un en charge du pôle Insertion (le devenir universitaire et professionnel des étudiants) et Condition de vie, (la condition de vie économique et sociale de la population étudiante : hébergement, conditions de travail, financement des études...), l'autre du pôle de la Vie scolaire (le processus d'orientation des étudiants au sein de l'Université de Toulouse : abandon, taux de réussite...). Tous deux sont chargés de la conception des études statistiques. Le premier réalise et analyse les enquêtes statistiques sur l'insertion professionnelle des étudiants, tandis que le second exploite les bases de données relatives à la population étudiante de l'université. Il s'agit, à partir de là, de décrypter, à l'aide d'indicateurs sociodémographiques, la population étudiée (nationalité, série au baccalauréat, statut de boursier, âge au baccalauréat).

L'étude de l'OVE à Toulouse 1 a pu être réalisée grâce à un croisement de méthodes. Ainsi, l'ambition d'ouverture à la multidimensionnalité constitue-t-elle le cadre épistémologique de notre recherche. Cela suppose la revendication d'un certain éclectisme méthodologique, qui passe par l'emploi de techniques d'observation et de traitement des données distinctes et complémentaires ${ }^{4}$ : les entretiens, individuels ou collectifs, l'observation participante ${ }^{5}$ et l'analyse documentaire ${ }^{6}$. C'est à partir de ce faisceau méthodologique que nous avons pu dégager l'importance de la dimension "territoire " dans l'opposition entre les OVE et le Ministère. Quelles sont donc cette prégnance territoriale et l'utilisation qui en est faite au coeur du conflit?

Nous nous interrogerons tout d'abord sur les normes de production territorialisée des résultats statistiques. Puis, à partir de là, nous tenterons d'analyser le processus d'opposition au projet IPSES en décrivant les étapes du conflit, à la lumière de la revendication des particularités locales par les OVE, soulignant, par ailleurs, le passage

+ Edgar Morin, "La démarche multidimensionnelle ", Sociologie, Paris, Seuil, coll. "Points ", 1994, p. 215-230.

Principalement à la $3^{\mathrm{c}}$ réunion nationale des OVE, qui s'est tenue à Toulouse les 20 et 21 novembre 2006 à la Manufacture des tabacs, amphithéâtre $\mathrm{H}$.

Surtout des productions respectives de l'OVE et du Portail étudiant. 
d'un conflit méthodologique à un enjeu de repositionnement organisationnel.

\section{Des arguments statistiques au profit d'une logique de territoire : de la description d'un système anomique à la recherche de référents méthodologiques unifiants}

Bruno Latour et Steve Woolgar expliquent que les travaux réalisés par les chercheurs sont fortement imprégnés de leur environnement culturel $^{7}$ : croyances, savoir-faire, histoire mythifiée et fondatrice du laboratoire...

En nous interrogeant sur le processus de construction des indicateurs statistiques par les membres de l'OVE de Toulouse 1, nous souhaitons élargir cette première approche en l'abordant sous un angle différent : celui de la prégnance du territoire dans les analyses réalisées par les statisticiens de ce même OVE (encastrement territorial des normes chiffrées, construction subjective par une identification symbolique au territoire). Au cours de notre réflexion, nous avons décidé de caractériser cette inscription territoriale par l'expression "normes latentes ", car nous souhaitions analyser la prégnance de principes implicites, environnants, diffus, sous-jacents, tels que l'influence du territoire, au sein des travaux des membres de l'OVE.

\subsection{Encastrement territorial des indicateurs statistiques ou illusion d'objectivité}

\subsubsection{Encastrement territorial et statistiques : paradoxe méthodologique?}

Parler d'encastrement territorial des normes statistiques peut sembler paradoxal. Dans quelque région française que ce soit, un taux d'insertion se calculera toujours de la même façon, comme un rapport entre le nombre d'étudiants ayant trouvé un emploi au cours des cinq années suivant leur sortie du système universitaire et le nombre total des

7 Bruno Latour et Steve Woolgar, La vie de laboratoire. L a production des faits scientifiques, Paris, La Découverte, 1996. 
sortants universitaires durant cette même période. De ce point de vue, les données statistiques chiffrées valent souvent caution d'objectivité, voire argument d'autorité, pour celui qui les emploie.

Mais, les indicateurs statistiques, en tant qu'effort de construction d'une intelligibilité du réel, n'en sont jamais l'exacte ou l'exhaustive retranscription. Ainsi, deux taux de chômage relatifs à deux pays distincts permettent, certes, de rendre compte avec impartialité de conjonctures économiques différentes, mais n'ont de valeur comparative que s'ils sont insérés dans les substrats sociaux et institutionnels qui leur confèrent leurs significations relatives. Les travaux d'Alain Desrosières et Laurent Thévenot ${ }^{8}$ sont précurseurs dans ce sens. Dans le cadre d'une analyse sociohistorique des catégories socioprofessionnelles (CSP), les auteurs mettent en exergue la prégnance des représentations politique, scientifique et cognitive au sein du processus de construction des CSP. Ils développent, par la suite, le problème statistique de la définition de l'identité sociale déterminant ces dernières, ainsi que la dimension politique de leur production par le biais de la participation des partenaires sociaux. Par ailleurs, à la lumière des travaux de Christian Topalov, les outils statistiques apparaissent comme " construits en fonction d'objectifs administratifs et de représentations savantes de la société, mais $[\ldots]$ ils doivent aussi, pour produire des chiffres et être utilisables, composer avec les représentations et les pratiques communes" $"$.

Topalov démontre bien que la distinction entre population active et population inactive a pris des chemins différents en France, en GrandeBretagne et aux États-Unis pendant plusieurs décennies, les dichotomies respectives reposent sur un critère commun : celui de la rémunération. Ainsi, la statistique ne peut-elle être appréhendée sociologiquement de manière analytique en tant que discipline extraite de son environnement; elle doit l'être comme un système écologique.

* Alain Desrosières et Laurent Thévenot, Les catégories socioprofessionnelles, Paris, La Découverte, 1996.

" Christian Topalov, «Une révolution dans les représentations du travail. L'émergence de la catégorie de la population active au XIXe siècle en France, en Grande-Bretagne et aux Etats-Unis ", Revue française de sociologie, vol. 40, $\mathrm{n}^{\circ} 3$, Paris, juillet-septembre 1999 , p. 446. 
De la même manière, cette influence environnementale se pose dans le cas des indicateurs statistiques de l'insertion étudiante. Ce qu'il s'agit de pondérer, et qui se trouve en même temps tégulièrement omis dans de nombreuses analyses, ce sont les influences des caractéristiques locales sous-jacentes, telles que le contexte socioculturel propre à l'université fréquentée ou le dynamisme des marchés du travail environnants, de telle sorte que les données de l'insertion ne valent jamais ex nibilo, mais au contraire comme outils à fonction heuristique. Si, comme nous le savons, "la carte n'est pas le territoire ${ }^{10}$ ", elle n'en demeure pas moins un outil pertinent pour qui sait s'en servir.

\subsubsection{Légitimité théorique d'une prise en compte des contextes territoriaux}

La notion d'encastrement (embeddness), ainsi que nous l'employons, s'appuie sur les développements de la sociologie économique, notamment dans sa dimension cognitive ${ }^{11}$. Cette notion nous semble, à ce titre, potentiellement féconde appliquée au cas concret des indicateurs statistiques de l'insertion professionnelle étudiante. Ces derniers, à l'image des marchés dont la constitution et le fonctionnement ne résultent jamais de la répétition systématique de lois dites naturelles, s'encastrent dans des environnements spécifiques dont il s'agit d'expliciter les caractéristiques avant de hasarder quelque hypothèse comparative que ce soit.

Conceptuellement, cette notion d'encastrement des normes chiffrées relatives à la vie étudiante n'est pas neutre et implique une redéfinition partielle de la notion de système universitaire. Selon Edgar Morin, le système est une « unité globale organisée d'interactions entre éléments, actions ou individus ${ }^{12} \%$. Cette définition présente le grand intérêt cognitif d'une focalisation sur les relations entre les constituants, plutôt que sur la nature de ces composants pris indépendamment. Or, un système, dans notre perspective, n'existe pas in abstracto, en dehors des

\footnotetext{
11 Alfred Korzybski, Unecarte n'est pas le territoire. Pralégomènes aux systèmes non-aristotéliciens et à la sémantique générale, Paris, L’Éclat, 1998.

"Paul Di Maggio et Sharon Zukin, Structures of capital: The social organization of the economy, Cambridge, Cambridge University Press, 1990.

12 Edgar Morin, La méthode. La Nature de la nature, Paris, Seuil, 1977, p. 9.
} 
considérations territoriales structurant son organisation ainsi que son évolution ré-organisatrice. Parler de système local d'enseignement supérieur interdit ainsi d'envisager une grille d'analyse uniforme, transposable quel que soit le contexte considéré, et impose a contrario une attention accrue pour les mécanismes d'identification et de réappropriation territoriales. D'un point de vue sémantique, cela nous conduit à préférer le terme de territoire à celui d'environnement dans l'étude de la capacité auto-organisatrice du système à élaborer lui-même les informations et les symboles nécessaires à son fonctionnement.

1.2. D'une nouvelle approche de la notion de territoire à la constitution d'une norme latente

\subsubsection{Identification symbolique aux territoires}

Pareil constat nous invite à repenser la question du territoire à l'aune des travaux subtils du géographe Michel Roux. Selon ce dernier, la conception traditionnelle du territoire, notamment véhiculée par les experts de l'aménagement, repose sur un présupposé ontologique faisant de l'espace une réalité en soi. Il en découle une méthode d'approche de type analytique fondée sur un schéma en trois temps :

- une décomposition du territoire en surfaces de distribution, construites à l'aide de grands indicateurs statistiques (densité, espérance de vie, PIB par habitant),

- une superposition de ces surfaces, et

- l'établissement d'une lecture croisée des structures récurrentes, dont on cherche à rendre compte de la logique, en mobilisant des modèles explicatifs, légitimés par des lois d'engendrement, importées des mathématiques ou de la physique ${ }^{13}$.

Cette étude d'inspiration cartésienne constitue le territoire en tant que réalité analysable objectivement, en tant que portion géométrique porteuse de ressources ou de potentialités, possédant des frontières identifiables, de telle sorte que les différents territoires existant objectivement se juxtaposent les uns aux autres sur un ensemble géographique

13 Michel Roux, Inventer un nouvel art d'babiter. Le ré-enchantement de l'espace, Paris, L'Harmattan, 2002. 
donné. De cette conception découle une prétention à l'expertise rationnelle et objective du territoire, la légitimité de l'aménagement reposant sur le diagnostic des aménageurs en fonction d'une norme globale d'intérêt général. En ce sens, le rapport Thouret de 1789, projetant un découpage départemental géométrique, constitue un exemple notable de la prégnance de ces présupposés ontologiques, en ce qu'il s'agissait d'une uniformisation de l'espace national en fonction d'une grille d'analyse homogène et transposable quel que soit le contexte considéré ${ }^{14}$.

A contrario, la définition que propose Roux du territoire repose sur deux hypothèses alternatives, fondatrices d'une nouvelle géographie ouverte à la multidimensionnalité.

La première constitue une mise en pratique de l'hypothèse phénoménologique telle qu'explicitée par Jean-Louis Le Moigne dans Les Épistémologies constructivistes ${ }^{15}$. Il s'agit de montrer que le territoire n'existe pas tant en soi, objectivement, que par soi, à travers nos représentations ${ }^{16}$. Dès lors on ne peut plus parler du territoire mais des territoires, dans le sens où il ne s'agit plus d'expertiser une réalité connaissable, mais d'élucider notre propre rapport aux choses, constituant une réalité par soi.

La deuxième hypothèse que développe Roux dans sa redéfinition des territoires est l'hypothèse téléologique. Cette téléologie souligne la capacité du vivant à finaliser son action, sa faculté autonome à donner un sens singulier à son environnement : l'espace habité existe par soi, mais également pour soi. Cela suppose une appropriation subjective des territoires par les individus connaissants. Cette appropriation relève de l'ordre du métaphorique, de l'onirique, dans la mesure où notre rapport individuel à l'espace est un rapport d'intimité, marqué par l'expérience personnelle, la culture, l'histoire des territoires en question. Roux parle

14 Pascal Roggero, "Entre représentations hiérarchiques et pratiques complexes : les paradoxes de la décentralisation française ", Nouvelles perspectives en sciences sociales, vol. $1, \mathrm{n}^{\circ} 1,2005$, p. 63-94.

15 Jean-Louis Le Moigne, Les Épistémologies constructivistes, Paris, PUF, 1995.

16. «L'environnement n'est pas un ensemble de données objectives mais le fruit d'une construction de notre cil et de notre esprit ", Michel Roux, op. cit., p. 24. 


\section{NPS\$, VOLUME 3, NUMERO 2, 2008}

ainsi d'enchantement de l'espace provenant du «paradoxe qui donne à la proximité des lieux aimés la faculté de nous transporter ailleurs ${ }^{17}$ \%.

À partir de cette nouvelle définition du concept territorial, qui fonde l'expérience individuelle comme critère d'identification et de compréhension, il devient possible d'envisager l'université non plus seulement selon son inscription spatiale, mais comme constituant elle-même un territoire ouvrant à une identification symbolique de la part de ses membres. Ce mécanisme de retraduction toujours singulier, faisant de la géographie non plus une affaire d'experts, mais au contraire «l'œuvre de tous ${ }^{18}$, repose sur la notion d'okéiosis : celle-ci, selon Roux, constitue la façon primordiale que nous avons de nous approprier intimement nos espaces en nous y identifiant à toutes les échelles (corps, bureau, entreprise, maison, rue, regard porté sur les êtres et sur les choses). Les propos recueillis auprès des étudiants de Toulouse 1, comme auprès des membres de l'observatoire, témoignent de la pertinence d'une réflexion en terme d'okéiosis, laquelle nous invite à rétablir la subjectivité des perceptions individuelles comme matériau sociologique, et réhabilite de la même manière l'assertion des sociologues américains William Isaac Thomas et Dorothy Swaine Thomas selon lesquels : If men define situations as real, they are real in their consequences ${ }^{19}$.

L'objectif de cette partie consisterait donc en une explicitation de l'okéiosis des membres des observatoires et de la manière dont il se traduit concrètement dans le cadre de normes statistiques partagées. Or, le problème d'ordre méthodologique qui se pose dans notre situation est celui de parvenir à synthétiser de manière collective des mécanismes singuliers d'identification. Ce rassemblement nous semble pourtant nécessaire d'un point de vue théorique. L'honnêteté scientifique nous impose néanmoins de considérer avec prudence une généralisation effectuée à partir de propos recueillis de manière éparse et qui ne valent que comme manifestation de rapports au monde nécessairement particuliers. Cependant, la participation à la $3^{\mathrm{e}}$ réunion nationale des

17. Conférence donnée le 17 novembre 2005 dans le cadre de la maîtrise (Master) II SCAT (Systémique complexe appliquée aux territoires) de l'université Toulouse 1.

18 Ibid., p. 25.

1.) Thomas, William Isaac et Dorothy Swaine Thomas, The cbild in America: Bebavior problems and programs, New York, Alfred A. Knopf, 1928, p. 572. 


\section{GEOFFREY CARRÈRE... / OBSERVATOIRES DE LA VIE ÉTUDIANTE...}

$\mathrm{OVE}^{20}$ nous. a permis de croiser les différentes représentations de l'espace géographique des membres des observatoires de la vie étudiante. Ainsi, avons-nous pu dégager, par la suite, au sein de la singularité des représentations, un référent commun : l'importance des caractéristiques locales.

\subsubsection{Une norme latente structurant l'activité des OVE : ne pas dénaturer les contextes locaux !}

De prime abord, notre insistance sur l'importance des caractéristiques locales dans le travail des experts des OVE peut paraître tautologique dans la mesure où, pour chaque observatoire d'université, la Présidence de l'établissement d'implantation constitue leur unique tutelle hiérarchique en même temps que leur principal commanditaire. "Nous on est d'abord un service d'aide à la décision interne ", nous confie ainsi la responsable du pôle vie scolaire ${ }^{21}$ de l'OVE de Toulouse 1 . À ce titre, l'OVE produit quasi-exclusivement ses analyses à la demande et à la destination du recteur d'université ou des directeurs d'unités de formation et de recherche (UFR). La marge d'initiative quant à la définition des sujets de travail semble dès lors très limitée, ainsi que le résume la même responsable :

Moi j'aimerais questionner les sortants non diplômés, pour voir ce qu'ils sont devenus trois ou cinq ans après leur sortie de l'université, mais la Présidence n'est pas intéressée. Les étudiants qui ne sont plus chez eux, ça ne les intéresse $\operatorname{pas}^{22}$.

Dans la pratique quotidienne, cependant, les commandes émanant des instances dirigeantes de l'université ne représentent qu'une partie de l'activité des responsables de pôles, d'ailleurs marquée dans leurs propos par une certaine dimension routinière. Ces mêmes responsables réalisent ainsi, de leur propre initiative, des enquêtes de cohortes tous les deux ans, afin d'approfondir les analyses usuelles par une perspective diachronique. De la même manière, ayant la plupart du temps à « interpréter le

\footnotetext{
20 Tenue à Toulouse les 20 et 21 novembre 2006 .

21 Entretien du 15 novembre 2006 à 10 h 45 dans le bureau de la responsable du pôle vie scolaire. 
silence $^{23}$ » des instances dirigeantes, l'observatoire de Toulouse 1 est décrit par le responsable du pôle insertion et condition de vie comme un service en constante "veille technique ${ }^{24}$ ». Il évoque, à ce titre, un projet d'étude commune sur les conditions de vie étudiantes, mené à la fois par les Universités de Toulouse 1, 2 et 3 .

«C'est nous qui allons susciter la demande [...] L'initiative nous revient à nous alors qu'elle devrait être institutionnalisée. ${ }^{25}$ " Pour autant, et en dépit de cette marge d'initiative relativement tangible, la prise en compte des caractéristiques locales demeure une ligne directrice véritablement prégnante du travail quotidien des statisticiens des OVE des trois universités toulousaines. La principale difficulté décrite au sujet de la construction de ces analyses statistiques concerne le nécessaire encastrement des indicateurs employés. Contrairement à l'idée répandue selon laquelle les chiffres constitueraient une mesure objective de la réalité, la responsable du pôle vie scolaire estime que «les chiffres ont leur histoire", que " chacun désigne des ensembles différents selon les université ${ }^{26} »$. De ce fait, pour les statisticiens des OVE, une autre compétence se greffe à la simple maitrise des indicateurs, à savoir la connaissance du terrain, du contexte multidimensionnel concret, sur lequel ces chiffres prennent sens :

Celui qui ne connait pas l'UT1 mais qui est béton en statisticues ne peut pas faire ce boulot. Il faut savoir que tel diplòme a été créé en telle année, à quelle catégorie il appartient. Par exemple le Deug MASS est considéré par le ministère comme faisant partie des Sciences de la Matière. Or, ici il est affilié à la Faculté de Sciences économiques. C'est juste qu'il faut le savoir, ça aurait pu appartenir à une autre UI’R. Ça c'est la connaissance du terrain ${ }^{27}$.

2.3 Entretien du 27 septembre 2006 à 9 h 30 dans le bureau du responsable du pôle insertion et condition de vie.

24 Entretien du 15 novembre 2006 à 9 h 30 dans le bureau du responsable du pôle insertion et condition de vie.

25 Ibid.

26. Entretien réalisé le 15 novembre 2006 à $10 \mathrm{~h} \mathrm{45,} \mathrm{op.} \mathrm{cit.}$

27 lbid. 
En l'occurrence, la spécificité du terrain considéré s'exprime ici en termes de convention, face au constat d'une relative indécidabilité : «ça aurait pu appartenir à une autre $U F R^{28}{ }^{2}$.

Cette prise en compte de l'encastrement des indicateurs implique, de la part des membres de l'OVE, un effort accru de prudence dans leur élaboration et leur manipulation. "Il faut toujours accompagner un indicateur de sa définition ${ }^{29} "$, résume ainsi la responsable du pôle vie scolaire, renchérissant par une anecdote expliquant que, dans les premiers temps, elle ne précisait pas dans ses comptes rendus la date d'étude, la population considérée ou la durée d'observation :

Il faut toujours préciser ce genre d'informations, sinon on ne peut rien comprendre aux chiffres. C'est justement ce qui manque dans la plupart des articles, et surtout dans le Portail étudiant. Pour moi, la partic méthodologique représente environ la moitié du temps de travail consacré à une étude ${ }^{31}$.

Cette relative convergence de préoccupations, indépendamment des distinctions en termes de parcours et de préférences individuelles, constitue-t-elle une spécificité toulousaine ou est-elle potentiellement applicable à l'ensemble du groupe des OVE?

Pareil questionnement semble légitime par souci de généralisation scientifique, et nécessaire compte tenu du projet d'envisager, dans notre seconde partie, le caractère collectif de l'opposition des OVE au Portail étudiant. Or, pour des raisons de faisabilité, notre terrain s'est concentré sur l'OVE de l'Université de Toulouse 1, et envisager une réponse exhaustive à cette question aurait imposé d'étendre l'observation menée aux OVE d'autres villes universitaires françaises. Un début de piste s'est néanmoins présenté à nous, lorsque s'est tenue à Toulouse, les 20 et 21 novembre 2006 , la $3^{\mathrm{e}}$ réunion nationale des OVE, en présence notamment de Guillaume Houzel, président de l'observatoire national, Fabrice Nicol, promoteur du projet IPSES auprès de la DEPP, et d'une trentaine de représentants d'observatoires de région ou d'université. Ce fut l'occasion, au terme de trois mois d'observations toulousaines, d'esquisser la comparaison entre nos premières conclusions et les 
pratiques, les parcours ainsi que les préoccupations d'autres cellules locales.

De ce point de vue, la prise de parole d'Alain Frickey, sociologue chargé de recherches au CNRS et membre de l'OVE de Nice-Sophia Antipolis, lors de la première conférence du lundi 20 novembre 2006, a semblé résumer un sentiment général en même temps qu'elle a suscité l'approbation d'une majorité des participants à la réunion, qu'ils soient représentants d'observatoires ou du Service commun universitaire d'information et d'orientation (SCUIO) :

Le contexte universitaire est particulier pour chaque région et demande des enquêtes spécifiques avec des méthodes singulières. Le cadre national nécessite une autre méthode et d'autres enquêtes. Le portail est peu clair sur ces précautions; il a un rôle hybride ${ }^{31}$.

Au-delà, donc, de l'éclatement institutionnel et statutaire caractérisant les différentes structures d'observatoires ainsi que leurs membres, et que Houzel, président de l'observatoire national de la vie étudiante, résume en parlant d'« objet non identifié dans la hiérarchie universitaire ${ }^{32} »$, une même préoccupation intellectuelle, celle de la singularité des contextes locaux, structure des publications éparses et contribue à l'unité du système, fondant ainsi la légitimité de même que la dynamique de l'action collective menée contre le projet IPSES.

\section{Des arguments organisationnels au profit d'une logique de pouvoir?}

Bien que marqués par une forte hétérogénéité, tant dans leur genèse que dans leur fonctionnement organisationnel, les observatoires locaux sont parvenus à développer un corpus relativement unifié de règles statistiques, s'exprimant sous la forme de précautions relatives au traitement et à la diffusion des informations chiffrées de la vie étudiante. Ces précautions ne suffisent cependant pas à définir un corps de métier cohérent : tout d'abord, en raison de l'absence de formalisation, puis parce que ces normes ne leur sont pas propres, mais découlent plutôt

$3^{\text {e }}$ réunion nationale des $\mathrm{OVE}$ tenue à Toulouse les 20 et 21 novembre 2006 à la Manufacture des tabacs, amphithéâtre $\mathbf{H}$.

32 Ibid. 
d'un background statistique englobant, hérité des sciences économiques et sociales.

Les normes quantitatives préexistantes font, par contre, l'objet d'une réappropriation singulière de la part des statisticiens des OVE, par l'attention portée aux contraintes d'encastrement des indicateurs employés, de telle sorte qu'en l'absence de référents univoques, il est néanmoins possible de distinguer un corps de préoccupations convergentes, formulées localement, mais susceptibles de servir de socle à une délimitation discriminante des pratiques des observatoires.

C'est cette attention portée à l'inscription des indicateurs, au sein de leur contexte territorial d'origine, qui rend d'autant plus contestable, aux yeux des OVE, l'action de centralisation et d'uniformisation menée par le Ministère par le Portail Étudiant. Selon la responsable du pôle vie scolaire :

Les OVE ont été créés pour éviter de greffer des données nationales sur des contextes régionaux, pour gagner en proximité dans la connaissance du milieu étudiant. Aujourd'hui on assiste au schéma inverse avec une forte volonté d'harmonisation de la part du Ministère. C'est oublier là qu'on a conféré de l'autonomie aux universités ${ }^{33}$.

2.1. Antagonismes méthodologiques entre les OVE et le Portail étudiant

\subsubsection{Fondements du projet IPSES}

Le projet IPSES, initié par le Ministère, aborde une dimension supplémentaire de l'activité traditionnelle des OVE, à savoir la diffusion des données chiffrées auprès du public étudiant. Les OVE des universités se définissent ainsi comme des services d'aide à la décision interne, réservant aux SCUIO locaux le soin de l'orientation et du conseil étudiant à partir des indicateurs qu'ils auront contribué à élaborer.

De ce point de vue, le projet de Portail étudiant n'apparaît en aucun cas contradictoire avec l'activité des observatoires, mais semblerait plutôt complémentaire de celle-ci. Le responsable du pôle insertion et condition de vie reconnait d'ailleurs une certaine pertinence au principe de ce projet, en tant qu'effort de centralisation de l'information relative

33 Entretien réalisé le 15 novembre 2006 à 10 h 45 dans le bureau de la responsable du pôle vie scolaire. 
aux différents cursus d'enseignement supérieur. "Sur le principe du Portail on est d'accord ${ }^{34}$ ».

Ce sont, par contre, les modalités concrètes de construction de l'information qui semblent se révéler conflictuelles, de mème que les justifications apportées à cet effort de communication. D'emblée, le même responsable dresse l'analyse d'un projet reposant sur un implicite contestable, selon lequel la connaissance permet une orientation cohérente. Tout se passerait comme si, conformément à l’analyse économique traditionnelle, l'acteur social - en l'occurrence l'étudiant - se chargeait de compiler l'ensemble des informations relatives à l'éventail de ses orientations possibles et s'efforçait ensuite de les traiter de manière synoptique afin de définir un choix d'étude optimal. Or, comme le reconnait le responsable, une orientation n'est jamais totalement objectivable ni cohérente, car elle repose davantage sur des affinités, des opportunités et des antipathies, que sur une mise en adéquation des moyens et des fins en fonction de l'information disponible.

Dans ce contexte d'incertitude radicale, les actions entreprises au jour le jour par les étudiants témoignent plus d'une nécessité de bricolage que d'un effort d'optimisation rationalisatrice. En ce sens, nous serions tentés d'évoquer des processus décisionnels étudiants sous l'angle d'un " modèle de la poubelle " s'effectuant en situation de rationalité limitée. À partir de la remise en cause, par Herbert A. Simon ${ }^{35}$ et Leon Festinger ${ }^{36}$, des trois postulats fondateurs de la théorie de la rationalité absolue (acteurs omniscients, dotés d'une capacité synoptique de traitement de l'information, et doués de préférences claires et hiérarchisables), les sociologues Michael D. Cohen, James G. March et John $P$. Olsen ${ }^{37}$ analysent l'anarchie décisionnelle existant au sein d'une

34 Entretien réalisé le 27 septem bre 2006 à 9 h 30 dans le bureau du responsable du pôle insertion et condition de vie.

35 Herbert A. Simon, Models of bounded rationality: Empirically grounded economic reason (Vol. 3), Cambridge, MIT Press, 1997.

3. Leon Festinger, A theory of cognitive dissonance, Stanford, Stanford University Press, 1957.

37. Michael D. Cohen, James G. March et John P. Olsen, "A garbage can model of organizational choice ", Administrative Science Quarterly, vol. 17, 1972, p. 1-25. 
organisation. Il s'agit de souligner la difficulté à prévoir les effets d'une nouvelle décision, ou de justifier une nouvelle décision par les effets qu'elle est censée avoir. Dans ce cas, une organisation apparaît comme le produit de la rencontre entre trois flux (flux de rencontres, flux de problème, flux de solution). La norme guidant ainsi la décision devient non plus optimale mais seulement "satisfaisante" (au sens de Simon). De la même manière, il nous a semblé possible d'analyser les comportements étudiants comme résultant de ces rencontres fortuites, de ces opportunités improbabilisables, leur conférant un caractère dialogique d'actions organisées-anarchiques.

Selon le même responsable, l'enjeu de ce projet de Portail Étudiant serait donc plutôt politique, en tant que réponse face à la crise du contrat première embauche. «Le Portail étudiant, c'est surtout un gros affichage politique ${ }^{38} » . \AA$ A partir de ce constat, le projet a été mené dans la précipitation et présente deux écueils majeurs.

- Tout d'abord, l'absence de concertation entre le Ministère et les différents OVE. La publication des résultats de l'OVE sur le Portail étudiant suppose l'emploi du logiciel IPSES. Ce dernier impose une nomenclature précise, en termes de définition des diplômes, qui ne correspond pas à celle de l'université Toulouse 1. Dès lors, de nombreux diplômes sont classés dans une catégorie qui ne correspond pas forcément aux perspectives de son enseignement.

- Il s'agit ensuite d'une absence de précautions méthodologiques dans la présentation des chiffres, et qui confine à la «malhonnêteté intellectuelle ${ }^{39} \%$.

2.1.2. Opérationnalisation des résultats nationaux : entre transparence et laxisme

La transparence : c'est le mot d'ordre de ce portail dédié, aux étudiants bien sût, mais également à tous ceux qui veulent mieux trouver leur chemin dans notre système d'enseignement supérieur. La transparence, c'est d'abord permettre à chacun de choisir en toute connaissance de cause son orientation, étape importante du parcours étudiant ${ }^{40}$.

38 Entretien du 27 septembre 2006 à 9 h 30, op. cit.

39) $3^{\text {c }}$ réunion nationale des OVE, op. cit.

4t Le Portail Étudiant, Ministère de l'Énseignement supérieur et de la Recherche, http://www.etudiant.gouv.fr/, consulté le 8 octobre 2006. 
Ces mots sont ceux par lesquels s'ouvre le site Web du Portail étudiant et ils nous apparaissent révélateurs quant aux modalités sociocognitives sous-jacentes à l'enjeu invoqué de transparence. Comme nous le rappellent Pascal Roggero et Claude Vautier :

La volonté de simplification qui apparait périodiquement dans les discours politiques repose sur une vision du système social inspirée des Lumières et de sa volonté de rationalisation du monde, de la vie, de la vie sociale. Or la simplification conduit à ignorer des aspects fondamentaux du système et de ses "métabolismes » et donc à appliquer de fausses solutions ${ }^{41}$.

Dans cette perspective, le critère de transparence est largement mis en avant par les promoteurs de ce projet, sans pour autant donner lieu à une définition synthétique du concept, sinon à la multiplication des témoignages de parcours étudiants potentiels. Or, celle-ci semble aller de pair, selon la responsable du pôle vie scolaire, avec un certain laxisme quant au traitement et à l'interprétation des données statistiques recueillies au niveau de chaque OVE.

Le Portail étudiant rassemble des données locales obtenues selon des méthodologies différentes, et les agglomère ensuite alors qu'elles sont incompatibles. Par exemple, certaines universités travaillent sur les étrangers hors Union Européenne, d'autres non, ou alors certaines fonctionnent sur des cohortes d'un an et demi, alors que d'autres travaillent sur des cohortes de trois ans. "C'est presque crapuleux, il leur faut des chiffres pour le portail afin de faire oublier l'histoire du CPE avant l'élection présidentielle ${ }^{42}$ ».

La conception de ce portail est décrite sur les modes de la précipitation et de l'absence de consultation, ce qui explique, aux dires de la responsable du pôle vie scolaire, le caractère statistiquement contestable des chiffres présentés par le Ministère par l'entremise du Portail étudiant. "Tout à coup il a fallu sortir le Portail en quelques mois. Et nous, on n'a pas du tout été consultés. Les cases qu'ils nous demandent de remplir n'ont aucune signification. [...] Ici on est les garants de

4) Pascal Roggero et Claude Vautier, “L'opacité du système politico-administratif français, essai de modélisation complexe ", Res-Systemica, n ${ }^{\circ}$, octobre 2002, p. 2.

t: Entretien du 3 novembre 2006 à $10 \mathrm{~h}$ dans le bureau de la responsable du pôle vie scolaire. 
l'honnêteté et de la validité des chiffres ${ }^{43}$ ». Plusieurs commentaires des responsables rencontrés semblent aller dans ce sens et dénoncent directement les indicateurs proposés par le Portail étudiant.

Les chiffres d'insertion de chaque cursus et de chaque université conduisent à une négation des spécificités locales, dans la mesure où il s'agit de comparer des catégories qui ne sont pas comparables.

"Pour l'insertion, on ne peut pas traiter toutes les données de la même manière, parce que les marchés de l'emploi dans le Nord Pas de Calais et en Midi Pyrénées ne sont pas du tout pareils. [...] Le Ministère tronque les savoirs, on nie les spécificités locales et géographiques ${ }^{44} »$.

Ces mêmes indicateurs, relatifs à l'insertion des étudiants sur le marché du travail, souffrent également d'une insuffisance liée au risque élevé d'erreur de première espèce ${ }^{45}$. C'est le cas, par exemple, de l'ancien DESS marketing de Toulouse 1, découpé depuis en quatre maitrises professionnelles. Chacune de ces maîtrises est spécifique, mais n'est étudié par le Portail étudiant qu'au travers d'un pourcentage unique supposant de computer des données brutes non comparables. De plus, sur ces classes à faibles effectifs, la validité des chiffres apparaît extrêmement sensible aux taux de réponse obtenus lors des enquêtes.

[Parlant des études portant sur des groupes aux effectifs réduit] Dès lors qu'on a des taux de réponse inférieurs à $70 \%$, les chiffres ne veulent plus rien dire. $\mathrm{Si}$, par exemple, j'ai deux individus seulement qui me répondent sur une classe de dix étudiants, et que leurs réponses donnent «oui » et "non ", ça voudrait dire que je dois mettre sur le Portail un taux d'insertion de $50 \%$. C'est à la fois faux, d'un point de vue scientifique, et malhonnête d'un point de vue déontologique ${ }^{46}$.

\subsubsection{Une critique récurrente : l'absence de concertation}

L'ensemble de ces critiques, d'ordre méthodologique, a fait l'objet d'intenses débats lors des réunions du 20 et 21 novembre 2006 entre Fabrice Nicol et les représentants des divers OVE. L'une d'entre elles,

\footnotetext{
43 Entretien du 15 novembre 2006 à 9 h 30, op. cit.

44 Entretien du 15 novembre 2006 à 10 h 45, op. cit.

45 On notera que le risque d'erreur de première espèce apparaît lorsque les liens de corrélations statistiques mis à jour s'appuient sur un nombre trop faible de cas concrets pour pouvoir être inférés à l'ensemble de la population.

46 Entretien du 15 novembre 2006 à 10 h 45, op. cit.
} 
en particulier, est apparue comme un leitmotiv de cette $3^{\text {e }}$ réunion nationale, à savoir le caractère "hautain " des services ministériels, qui ont initié le projet sans consultation des membres des OVE, et les ont ensuite assignés à remplir leurs propres grilles statistiques. Plus que la nature même de ces grilles statistiques imposées et les défaillances méthodologiques pointées du doigt par les membres des OVE, le ressort principal de leur opposition organisée résiderait dans un sentiment d'aliénation à l'égard des chiffres communiqués. "Nous ne souhaitons pas devenir de simples presse-boutons ${ }^{47}$ ", nous explique ainsi une statisticienne de l'observatoire de Lille II ayant participé à la réunion nationale des OVE.

Cette absence de concertation peut s'expliquer par la non-existence, avant novembre 2006, d'un interlocuteur unique représentant les observatoires; le caractère éclaté du système des OVE de France depuis leur création a exigé, de la part des services de la Direction de l'évaluation de la prospective et de la performance (DEPP), de contacter séparément chaque observatoire afin de solliciter les chiffres attendus. Cette démarche est cependant allée de pair avec une définition unilatérale des grilles statistiques, de leur construction et de leur définition. Là où les observatoires jouissaient jusqu'à présent d'une situation de monopole statistique, s'intercale désormais un nouvel acteur prétendant agir au nom de la transparence et de la cohérence à échelle nationale incarné par le Ministère à travers le Portail Étudiant.

Hasardons une hypothèse : fédérer dans le cadre d'une association loi 1901 les structures éclatées des OVE fut déjà tenté lors des deux premières réunions nationales de 2004 et 2005, mais sans succès. A contrario, deux heures de réunion furent nécessaires, le mardi 21 novembre 2006, pour fonder l'association nationale des observatoires de la vie étudiante, élire son président (Marc Boudier) ainsi que son conseil d'administration. L'apparente rapidité de cette procédure fut, certes, rendue possible par le constat d'une opposition partagée face à un adversaire identifié, le projet IPSES, mais il fallait également l'affirmation d'un consensus quant à une identité commune qui, nous l'avons

Entretien lors de la réunion' sur l'extranet et la faisabilité d'un portail des OVE, le mardi 21 novembre 2006, à $14 \mathrm{~h}$. 
vu, n'allait pourtant pas de soi. Cette identité, latente au niveau du partage de normes statistiques implicites, ne s'est véritablement révélée qu'à partir de la prise de conscience par les membres des OVE d'une menace quant à la définition de leur statut organisationnel. C'est ainsi qu'à la question sur la nature du conflit avec le Ministère, une responsable d'observatoire de Bordeaux affirme qu'il s'agit " [d']un conflit méthodologique, rien de plus ", avant de confesser que "les rôles étaient bien définis jusqu'à présent, nous on sait ce qu'on a à faire ${ }^{48} »$.

À partir d'une mise à jour des normes implicites structurant le travail quotidien des statisticiens des OVE, nous nous sommes ainsi efforcés de définir la nature du conflit organisé autour du projet de Portail étudiant. Mais au-delà de cette opposition relative à la construction des chiffres de l'insertion étudiante, la compréhension du phénomène de constitution des OVE en association exige de dépasser le caractère unilatéral d'une analyse exclusivement méthodologique, et impose de considérer également la répartition informelle des rapports de force existant entre projet ministériel d'uniformisation de l'information et services délocalisés d'aide à la décision universitaire. En ce sens, l'analyse d'un conflit né de la crainte des observatoites d'une marginalisation organisationnelle semble légitime quant à la construction des chiffres de l'insertion étudiante. Le projet IPSES aborde, cependant, une autre dimension tout autant sujette à polémique, à savoir la communication de ces mêmes résultats.

\subsection{Antagonismes liés à la diffusion des résultats}

\subsubsection{Importance du devenir des résultats}

Nous avons vu précédemment que la nature du conflit entre les OVE et le Ministère était d'ordre méthodologique autant qu'organisationnel. Or, il semble que cette opposition dépasse les simples frontières de la construction statistique. En ce sens, de nouvelles interrogations émergent. Celles-ci sont relatives à un tout autte domaine, celui de la

48 Entretien informel réalisé à la sortie de la réunion sur la construction des enquêtes triennales par l'Observatoire national de la vie étudiante, Toulouse, le 21 novembre 2006 , à $10 \mathrm{~h}$. 
vulgarisation des résultats. Daniel Jacobi entend par vulgarisation scientifique l'ensemble des procédés de communication en provenance du champ scientifique, conférant à cette notion une extension notable ${ }^{49}$. Elle désigne aussi bien la communication dont les scientifiques font usage entre eux, que la communication de leurs découvertes à ceux qu'elles peuvent concerner directement (les utilisateurs) ou indirectement (le grand public). Au coeur de la rivalité entre les OVE et le Ministère s'affrontent deux formes de vulgarisation générant une lutte autour du devenir de l'étude et, plus largement, autour de ce qui fait la spécificité des fondements et du fonctionnement de ces deux entités.

"Le gros problème n'est pas la construction mais la diffusion. Le problème de la vulgarisation est qu'il faut aller à l'essentiel sans trahir la complexité $e^{501}$. Cet énoncé du responsable du pôle insertion et condition de vie nous renvoie à l'article de Bernard Schiele « Publiciser la Science! Pour quoi faire? $»^{51}$ où il s'interroge sur la « mésestimation de la publicisation des sciences et des techniques » et sur le "manque chronique de connaissances scientifiques et techniques d'une large fraction du public $^{52} »$. Problème d'autant plus important que notre société est devenue une société du savoir "sans cesse plus complexe à déchiffrer $\left.^{53}\right)$.

Notre analyse se réalise au cœur de cette problématique actuelle et, plus particulièrement, autour de la question de la traduction de la science. Schiele et Jacobi expliquent la complexité de ce processus dans son rapport avec le public. «[L]es informations assimilées par un sujet proviennent d'une exposition aléatoire aux médias, aux conversations

49 Daniel Jacobi, L a communication scientifique : discours, figures, modeles, Grenoble, Presses universitaires de Grenoble, 1999.

5॥ Entretien réalisé le 15 novembre 2006 à 9 h 30 dans le bureau du responsable du pôle insertion et condition de vie.

51 Bernard Schiele, " Publiciset la science! Pour quoi faire?", dans Isabelle Pailliart (dir.), La publicisation de la science. Exposer, communiquer, débattre, publier, vilgariser, Grenoble, Presses universitaires de Grenoble, 2005, p. 11-52.

5. Ibid., p. 11.

5.3 Ibid. 
ou à toute autre forme d'échange ${ }^{54}$.» Or, c'est le récepteur qui donne un sens propre à l'information.

Ainsi, la diffusion des résultats détient une place centrale au sein du conflit entre les OVE et le Ministère. Elle est d'autant plus importante que l'université est devenue, depuis quelques années, un enjeu politique d'envergure tant sur le plan local que national avec les manifestations étudiantes contre le projet de contrat première embauche (CPE). Ce conflit se traduit donc par l'affrontement de deux mises en forme de diffusion s'articulant autour de deux stratégies divergentes répondant à des contraintes à la fois institutionnelles et environnementales singulières : la première vulgarisation, celle des OVE, basée sur la traduction de la complexité des phénomènes universitaires locaux, la seconde, celle du Ministère, fondée sur une analyse homogénéisatrice et rationnelle de ces mêmes phénomènes.

Il convient donc d'analyser les antagonismes méthodologiques actuels entre ces deux formes de vulgarisation.

\subsubsection{Affrontement de deux formes de vulgarisation}

Lors de la rédaction de ces travaux, le responsable du pôle insertion et condition de vie se heurte à une double contrainte relative à la présentation de ses résultats. Si nous reprenons la définition de l'expert établie par Philippe Roqueplo, "situé à l'interface de la connaissance et de la décision, l'expert scientifique, se trouve sur le versant connaissance de cette interface ${ }^{55} "$, la notion de vulgarisation apparaît prégnante dans le domaine de l'expertise. Ainsi, les experts statisticiens de l'OVE réalisent une activité de traduction en transposant leurs conclusions scientifiques en résultats d'aide à la décision universitaire. Pour cela, chaque production doit être vulgarisée à des fins de compréhension institutionnelle sans pour autant trahir la complexité du réel. En ce sens, le responsable du pôle insertion et condition de vie réalise un texte de

${ }^{54}$ Bernard Schiele et Daniel Jacobi, "La vulgarisation scientifique. Thèmes de recherche ", dans Daniel Jacobi et Bernard Schiele (dir.), Vulgariserla science. L e procìs de l'ignorance, Seyssel, Champ Vallon, 1988, p. 21.

55 Philippe Roqueplo, Entre savoir et décision, l'expertise scientifique, Paris, INRA, 9 avril 1997, p. 14. 
synthèse de quatre pages auquel il joint une analyse détaillée de cinquante ou soixante pages.

Le premier texte de vulgarisation a l'avantage d'être plus lisible, cependant il ne comporte pas de préconisations méthodologiques préalables à toute analyse statistiques. «Sur un boulot de quatre pages on va à l'essentiel, on décrit juste les données ${ }^{56}$. " Le second pèche par son exhaustivité : «Le problème est que personne le lit ${ }^{57}$. " Cependant, ce texte comporte toutes les précautions méthodologiques nécessaires ainsi que les analyses de comparaison des résultats d'année en année. "Le problème de la diffusion de l'information est liée à son contrôle $[\ldots]$ chacun fait sa propre utilisation ${ }^{58} "$.

Dès lors, afin d'assurer le contrôle de la diffusion des analyses et d'éviter leur utilisation dénaturée, le même responsable va mobiliser sa connaissance du système universitaire de Toulouse 1 afin d'adapter les résultats à la population à laquelle il s'adresse. En ce sens, ce responsable ne se définit pas comme une simple source d'information mais plutôt comme "une source d'information pour les vecteurs d'informations $"{ }^{59}$. Il construit donc la diffusion des résultats en fonction des services d'information, SCUIO par exemple, auxquels il s'adresse. Nous pouvons constater que l'activité vulgarisatrice des membres de l'OVE fait l'objet de multiples précautions à la fois méthodologiques et relatives à son mode même de diffusion par son adaptation à la population concernée. La vulgarisation mise en place est donc à mi-chemin entre la production scientifique et la prospective et elle fait l'objet d'un double encastrement environnemental relatif au contexte institutionnel et géographique. Elle impose, en ce sens, plusieurs mises en garde.

C'est autour de ce dernier point que le conflit entre les OVE et le Ministère s'articule. Le reproche essentiel fait au Portail étudiant est l'absence de préconisations suffisantes permettant de relater avec précision le contexte local. En fait, le projet IPSES se réalise au sein

56 Entretien du 3 novembre 2006, à 11 h 30, dans le bureau du responsable du pôle insertion et condition dé vie.

57 Ibid.

5. Ibid.

54. Eintretien du 3 novembre 2006 à 11 h 30, op. cit. 
d'une stratégie généralisatrice, nécessaire au plan national, à des fins de meilleure accessibilité publique opérée par logique de simplification.

\subsection{3. À la recherche d'un compromis de vulgarisation : l'enjeu des liens hypertextes}

La vive mobilisation des observatoires, renforcée par la création de l'Association nationale des OVE le mardi 21 novembre 2006 à 12 h, a amorcé la prise de conscience par les services de la Direction de l'évaluation de la prospective et de la performance (DEPP) des lacunes du projet. Ainsi, ce dernier a-t-il intégré les difficultés relatives à la comparabilité des résultats, à l'hétérogénéité des contextes et des méthodes statistiques mobilisées. De cette controverse est né le compromis, proposé par Fabrice Nicol, et présenté comme une "main tendue ", de renvoyer chaque résultat du Portail étudiant aux études originelles des observatoires concernés, par la création de liens hypertextes. En termes de vulgarisation, il s'agissait ainsi de conserver la grande rapidité d'accès à l'information, fondement du projet initial de portail national, tout en répondant aux critiques d'une simplification excessive par la possibilité, pour chaque étudiant, de pondérer les résultats consultés à partir des contextes spécifiques de chaque université.

$D^{\prime}$ autres mesures furent également proposées par le représentant de la DEPP, telles que la présentation des résultats de l'insertion sous forme d'effectifs plutôt que de pourcentages, ou encore la prise en compte des dénominations des filières propres à chaque université. Néanmoins, cette question de la création des liens hypertextes et du renvoi aux études des observatoires nous a semblé constituer la pierre angulaire du compromis patronné par Nicol, en raison notamment de la véhémence des débats suscités par cette proposition.

D'un point de vue strictement méthodologique, il s'agissait là d'une prise en compte des critiques des OVE qui aurait pu être en mesure de les satisfaire. La proposition fut cependant refusée, l'opposition au projet IPSES maintenue, et la perspective d'un projet alternatif et concurrent de portail des observatoires, conservée. À ce stade du conflit, il fut donc impossible pour le représentant ministériel de désamorcer le processus d'opposition organisée. En outre, le rejet de la " main tendue " de Nicol, en rapport à la multiplication des liens 
hypertextes au sein du Portail étudiant, semble confirmer notre hypothèse d'une opposition des OVE au projet IPSES au moins autant motivée par la crainte d'un repositionnement organisationnel dans le champ de la production des indicateurs statistiques de l'insertion étudiante, que par le constat des insuffisances méthodologiques du projet ministériel.

\section{Conclusion}

- Le conflit entre les OVE et le Ministère est d'ordre méthodologique?

- Oui, oui il est d'ordre méthodologique, méthodologique "i".

Le désaccord entre les OVE et le Ministère a été le socle de notre analyse. Ce contexte conflictuel a permis de mettre en exergue deux modèles de construction d'analyse statistique. D'une part, celui du Ministère répondant à des prescriptions homogénéisatrices et, d'autre part, celui des OVE revendiquant l'inscription de leurs études au cœur de contextes locaux particuliers et sollicitant, de ce fait, un savoir singulier exprimé ici dans les normes implicites d'encastrement territorial et de convergence des savoirs.

En ce sens, les OVE mobilisent une méthodologie singulière. Il s'agit de souligner la construction des résultats statistiques sous le prisme des données territoriales et de justifier leur positionnement subjectif dans la réalisation de leurs études. Cette orientation scientifique s'exprime aussi dans le paradoxe d'une association de savoirs hétérogènes, mais convergents, afin de bénéficier d'une meilleure lecture du " réel ". L'importance des liens hypertextes, au cceur du débat entre les OVE et le Ministère, participe de ce même mouvement de revendication des spécificités locales dans le cadre des analyses statistiques.

Précisément, les liens hypertextes auraient pu être un compromis permettant de sortir de cet antagonisme méthodologique, conciliant à la fois les revendications des OVE et les perspectives du projet IPSES. Pour autant ce ne fut pas le cas et, en dépit des déclarations contraires affirmant le caractère exclusivement méthodologique du conflit, ce refus semble aussi accompagner un mouvement de repositionnement organisationnel des OVE par rapport au Ministère.

(6) Entretien lors de la réunion sur l'extranet et la faisabilité d'un portail des OVE, le 21 novembre 2006 , à $14 \mathrm{~h}$, Toulouse. 
Même dans ces situations de dépendance et de contrainte, non seulement les hommes ne s'adaptent donc pas passivement aux circonstances, mais ils sont capables de jouer sur elles et ils les utilisent beaucoup plus souvent qu'on ne croit de façon active ${ }^{01}$.

Bien au-delà d'une attitude de subordination bienveillante, ou pour le moins neutre, attendue par les services de la DEPP des observatoires locaux, ces derniers ont su s'approprier ce projet de façon dynamique sur le mode de la contestation méthodologique. Mais le caractère unanime de cette opposition, bien que s'exprimant de manières distinctes, a aussi constitué le détonateur et le moteur d'une vaste transition systémique dont il est encore difficile d'envisager les effets. En tant que jeunes chercheurs en sociologie, nous sommes ainsi entrés dans la phase d'observation monographique des observatoires de la vie étudiante de Toulouse dans un contexte d'anomie organisationnelle marquée par l'éclatement et l'absence de coordination entre les observatoires. Les désirs de réformes structurelles étaient présents sans quoi la rapidité du processus de constitution en association lors du congrès national de Toulouse n'aurait probablement pas pu être possible ou l'aurait été de manière trop ponctuelle et insuffisamment relayée localement pour aboutir. Trois mois plus tard, l'association constitue une réalité administrative, dont il sera nécessaire d'étudier le fonctionnement avec plus de recul afin de mesurer attentivement l'ampleur de la transition observée au sein de cette recherche.

Bien au-delà des anticipations du Ministère, une initiative censée se substituer à l'hétérogénéité des observatoires locaux, dans la perspective d'une récupération politique des chiffres de la vie étudiante les a, de fait, institutionnalisés nationalement. Les OVE se sont repositionnés organisationnellement autour de la connaissance des spécificités locales donnant sens à leur travail. Ce rapprochement, qui a fait émerger un consensus identitaire d'une opposition commune, les a renforcés et les a positionnés en interlocuteur de poids face au Ministère.

61 Michel Crozier et Erhard Friedberg, L'acteur et le système. Contraintes de l'action collective, Paris, Seuil, 1977, p. 43. 


\section{Bibliographie}

Bourdieu, Pierre et Jean-Claude Passeron, Les Héritiers. Le's étudiants et la culture, Paris, Minuit, 1964, $192 \mathrm{p}$.

Cohen, Michael D., James G. March et John P. Olsen, "A garbage can model of organizational choice ", Administrative Science Quarterly, vol. 17, 1972, p. 1-25.

Crozier, Michel et Erhard Friedberg, L'acteur et le système. Contraintes de l'action collective, Paris, Seuil, 1977, 500 p.

Desrosières, Alain et Laurent Thévenot, Les catégories socioprofessionnelles, Paris, La Découverte, $1996,125 \mathrm{p}$.

Di Maggio, Paul et Sharon Zukin, S'tructures of capital: The social organization of the economy, Cambridge, Cambridge University Press, 1990, 461 p.

Festinger, L.eon, A theory of cognitive dissonance, Stan ford, Stanford University Press, 1957, $239 \mathrm{p}$.

Jacobi, Daniel, La communication sicientifique : discours, figures, modèles, Grenoble, Presses universitaires de Grenoble, 1999.

Korzybski, Alfred, Une carte n'est pas le territoire. Prolégomènes aux systèmes non-aristotéliciens et à la sémantique générale, Paris, L'Éclat, 1998, $204 \mathrm{p}$.

Iatour, Bruno, "Nouvelles règles de la méthode scientifique ", Ceras, Revue Projet, $\mathrm{n}^{\circ}$ 268, décembre 2001, URL: http://www.ceras-projet.com/index.php?id $=1868$.

Latour, Bruno et Steve Woolgar, La vie de laboratoire. La production des faits scientifiques, Paris, L.a Découverte, 1996, 299 p.

L.e Moigne, Jean-Louis, Les Épistémologies constructivistes, Paris, PUF, coll. «Que saisje? ", $1995,128 \mathrm{p}$.

Loubet del Bayle, Jean-Louis, Initiation aux méthodes des sciences sociales, Paris, L'Harmattan, 1998, 272 p.

Ministère de l'Enseignement supérieur et de la Recherche, Le Portail Étudiant, http://www.etudiant.gouv.fr/, consulté le 8 octobre 2006.

Morin, Edgar, La tête bien faite, Paris, Seuil, 1999, 153 p.

Morin, Edyar, «La démarche multidimensionnelle », Sociologie, Paris, Seuil, coll. "Points", 1994, p. 215-230.

Morin, Edyar, I a métjode. La Nature de la nature, Paris, Seuil, 1977, 399 p.

Roggero, Pascal, "Lntre représentations hiérarchiques et pratiques complexes : les paradoxes de la décentralisation française ", Nouvelles perspectives en sciences sociales, vol. $1, \mathrm{n}^{\circ} 1,2005$, p. 63-94.

Roggero, Pascal et Claude Vautier, "L'opacité du système politico-administratif français, essai de modélisation complexe ", Res-Systemica, $\mathrm{n}^{\circ} 2$, octobre 2002, URL : http://www.afscet.asso.fr/resSystemica/Crete02/Roggero.pdf.

Roqueplo, Philippe, Entre saboir et décision, l'expertise scientifique, Paris, IN RA, 9 av ril 1997, $111 \mathrm{p}$.

Roux, Michel, Inventer un nouvel art d'babiter. Le ré-enchantement de l'espace, Paris, L'Harmattan, coll. "Ingenium », 2002, 204 p.

Schiele, Bernard, "Publiciser la science! Pour quoi faire?", dans Isabelle Pailliart (dir.), La publicisation de la science. Exposer, communiquer, débattre, publier, vulgariser, Grenoble, Presses universitaires de Grenoble, 2005, p. 11-52. 


\section{GEOFFREY CARRÈRE... / OBSERUATOIRES DE LA VIE ÉTUDIANIE...}

Schiele, Bernard et Daniel Jacobi, "La vulgarisation scientifique. Thèmes de recherche", dans Daniel Jacobi et Bernard Schiele (dir.), Vulgariser la science. Le procès de l'ignorance, Seyssel, Champ Vallon, coll. “ Milieux ", 1988, p. 12-46.

Simon, Herbert A., Models of bounded rationality: Empirically grounded economic reason (Vol. 3), Cambridge, MIT Press, 1997, 479 p.

Thomas, William Isaac et Dorothy Swaine Thomas, The child in America: Behavior problems and programs, New York, Alfred A. Knopf, 1928, 586 p.

Topalov, Christian, "Une révolution dans les représentations du travail. L'émergence de la catégorie de la population active au XIX' siècle en France, en GrandeBretagne et aux États-Unis ", Revue frangaise de sociologie, vol. 40, $\mathrm{n}^{\circ} 3$, Paris, juilletseptembre 1999 , p. $445-473$. 